\title{
Conservatism, growth, and return on investment
}

\author{
Madhav V. Rajan · Stefan Reichelstein • \\ Mark T. Soliman
}

Published online: 15 May 2007

(C) Springer Science+Business Media, LLC 2007

\section{Erratum to: Rev Acc Stud \\ DOI: $10.1007 / \mathrm{s} 11142-007-9035-2$}

The Publisher apologizes for the errors in this article. Proposition 1, Eq. 3 is missing two " -1 ". The erroneous " + "' should also be deleted. Please see corrected version below:

Proposition 1 Conservative accounting implies:

$$
\operatorname{ROI}_{T}(\vec{\lambda})\left\{\begin{array}{l}
\geq r \text { if } \lambda_{t} \leq r \text { for all } 1 \leq t \leq T-1 \\
\leq r \text { if } \lambda_{t} \geq r \text { for all } 1 \leq t \leq T-1 .
\end{array}\right.
$$

Conversely, with liberal accounting, $\operatorname{ROI}_{T}(\vec{\lambda}) \leq r$ if $\lambda_{t} \leq$ for all $1 \leq t \leq T-1$, while $\operatorname{ROI}_{T}(\vec{\lambda}) \geq r$ for $\lambda_{t} \geq r, 1 \leq t \leq T-1$.

The online version of the original article can be found at http://dx.doi.org/10.1007/s11142-007-9035-2

M. V. Rajan $(\bowtie) \cdot$ S. Reichelstein · M. T. Soliman

Graduate School of Business, Stanford University, Stanford, CA, USA

e-mail: mrajan@gsb.stanford.edu

S. Reichelstein

e-mail: reichelstein@stanford.edu

M. T. Soliman

e-mail: msoliman@stanford.edu 